\title{
AVALIAÇÃO DO DESEMPENHO CATALÍTICO DE ANODOS PARA CÉlUlAS SOFC A BASE DE Ni SUPORTADO EM CeZr, CePr e CeNb NA REFORMA SECA DO METANO
}

\author{
R. O. da Fonseca, ${ }^{1,3}$, A. A. A. da Silva ${ }^{2,3}$, M. R. M. Signorelli1 ${ }^{1,3}$, R. C. R. Neto $^{3}$, F. B.
} Noronha $^{3}$,L. V. Mattos ${ }^{1}$, R. C. C. Simões ${ }^{1}$

${ }^{1}$ Universidade Federal Fluminense, Departamento de Pós Graduação de Engenharia Química

${ }^{2}$ Instituto Militar de Engenharia , Departamento de Pós Graduação de Química

${ }^{3}$ Instituto Nacional de Tecnologia, Divisão de Catálise e Processos Químicos

Email: lisiane@vm.uff.br

RESUMO - Neste trabalho, o desempenho catalítico de anodos a base de níquel suportados em $\mathrm{CeNb}, \mathrm{CePr}$ e CeZr foi avaliado na reforma seca do metano a 1073K. Os materiais foram caracterizados através de medidas de área específica, análises de difração de raios $X$, redução à temperatura programada e termogravimetria. Os resultados mostraram a formação da solução sólida para os catalisadores suportados em $\mathrm{CePr}$ e CeZr. Além disso, o material a base de CeZr apresentou o menor tamanho de cristalito de níquel metálico, o que levou a um maior valor de atividade inicial na reação de reforma seca. Entretanto, ao contrário do $\mathrm{Ni} / \mathrm{CeZr}$, o anodo a base de $\mathrm{CePr}$ não apresentou desativação significativa, com baixa formação de carbono, após a reação. Esse resultado foi atribuído à maior mobilidade de oxigênio da amostra contendo Pr, o que promove o mecanismo de remoção do carbono da superfície metálica.

\section{INTRODUÇÃO}

Um dos grandes desafios na exploração de petróleo na área do pré-sal está relacionado com o meio ambiente. O gás natural associado encontrado nas reservas do pré-sal é composto, principalmente, por metano e dióxido de carbono, os quais contribuem para o aumento do efeito estufa quando queimados nas plataformas (Gouveia, 2010). Dentro deste contexto, a utilização do gás associado na co-geração de energia elétrica e calor para a própria plataforma através de células a combustíveis do tipo óxido sólido (SOFC) pode ser considerada uma alternativa promissora para o aproveitamento deste gás (Ahmed e Krumpelt, 2001).

As células do tipo SOFC transformam energia química em energia elétrica e são compostas por um anodo, um catodo e um eletrólito sólido. O catodo é alimentado com ar e o anodo pode ser alimentado diretamente com um combustível (como hidrocarbonetos ou álcoois). No catodo, são produzidos os íons $\mathrm{O}_{2}{ }^{-}$, que são conduzidos até o anodo, através do eletrólito. No anodo, esses íons reagem com o $\mathrm{H}_{2}$ e o $\mathrm{CO}$, que são produzidos na reforma do combustível, gerando corrente elétrica (Sun e Stimming, 2007). No caso do uso do gás associado como combustível para células SOFC, o hidrogênio seria gerado nos anodos a partir da reforma do metano com $\mathrm{CO}_{2}$ (reforma seca do metano), ambos provenientes do próprio gás associado. Além da disponibilidade e baixo custo dos reagentes, o investimento total deste processo é menor, quando comparado à produção de hidrogênio e $\mathrm{CO}$ pela reforma a vapor do metano. 
Os materiais mais utilizados como anodos em células SOFC são a base de Ni/YSZ (Sun e Stimming, 2007; Ke et al., 2006). O Ni é responsável pela condutividade eletrônica e a atividade catalítica na reforma do combustível, enquanto que o suporte é o responsável pela condutividade iônica e estabilidade. Todavia, é necessário o uso de teores de Ni acima de $30 \%$ em volume e altas temperaturas para se seja obtida uma condutividade adequada. Porém, como o níquel é muito ativo para o craqueamento de hidrocarbonetos, teores tão altos deste metal levam à formação de grandes quantidades de coque, provocando a perda de atividade catalítica. Observou-se, também, que o uso de altas temperaturas contribui para a sinterização do suporte e, consequentemente, para o crescimento das partículas $\mathrm{Ni}$, o que causa a desativação do material (Sun e Stimming, 2007). Assim, anodos que apresentem teores mais baixos de Ni, suportes mais resistentes à sinterização e que apresentem boa atividade para a reforma seca do metano, são promissores para serem utilizados na reforma do metano com $\mathrm{CO}_{2}$ nas células SOFC.

No caso do teor de $\mathrm{Ni}$, alguns trabalhos mostraram que a preparação de anodos com baixos teores de metal ( 8-15\% em peso), usando métodos de preparo alternativos, permitiu a obtenção de materiais com boa condutividade em células alimentadas com $\mathrm{H}_{2}$ (Jasinski et $a l ., 2005)$ e com bom desempenho catalítico em reações de reforma do propano (Modafferi et al., 2008). Com relação ao suporte, alguns autores (Sun e Stimming, 2007) reportaram que o uso de materiais a base de $\mathrm{Ce}_{0,9} \mathrm{Gd}_{0,1} \mathrm{O}_{1,95}$ no anodo de células SOFC permitiu a obtenção de alta condutividade em menores temperatura. Além disso, a adição de dopantes como o $\mathrm{Zr}$ aumenta a estabilidade do $\mathrm{CeO}_{2} \mathrm{em}$ altas temperaturas e a capacidade de armazenamento de oxigênio do suporte, o que promove o mecanismo de remoção de carbono da superfície metálica (Mattos et al., 2002). Neste contexto, outros dopantes como os óxidos de metais terras raras ( $\mathrm{Nb}$ e $\mathrm{Pr}$ ), que são utilizados usualmente, poderiam ser adicionados ao $\mathrm{CeO}_{2}$ para serem testados e comparados com o suporte de $\mathrm{CeZrO}_{2}$.

Portanto, o objetivo deste trabalho foi avaliar o desempenho catalítico de anodos a base de $\mathrm{Ni} / \mathrm{CeZr}, \mathrm{Ni} / \mathrm{CeNb}$ e $\mathrm{Ni} / \mathrm{CePr}$, na reforma seca do metano.

\section{SEÇÃO EXPERIMENTAL}

Os catalisadores foram preparados usando-se o método hidrotérmico, seguido de impregnação úmida com o precursor de níquel (Modafferi et al., 2008). Inicialmente, foram obtidos os suportes $\mathrm{CeNb}$, CePr e $\mathrm{CeZr}$ (na proporção molar de cério/dopante de 9:1) utilizando-se Nitrato de Cério Amoniacal (IV), Nitrato de Praseodímio e de Zircônio e Oxalato de Nióbio como sais precursores. Após solubilização em água destilada, os precursores foram misturados e co-precipitados com hidróxido de amônio em excesso. Os precipitados obtidos foram transferidos para uma autoclave onde foram submetidos a um tratamento hidrotérmico a $453 \mathrm{~K}$ durante $4 \mathrm{~h}$. Em seguida, as amostras foram lavadas em água destilada até $\mathrm{pH} 7$ e submetidas a uma secagem em estufa a $358 \mathrm{~K}$, por $12 \mathrm{~h}$. Os suportes obtidos foram, então, calcinados a $573 \mathrm{~K}$, por $2 \mathrm{~h}$, sob fluxo de ar.

Após a calcinação, o $\mathrm{Ni}$ ( $18 \%$ em peso) foi adicionado aos suportes pelo método de impregnação seca, utilizando-se $\mathrm{Ni}\left(\mathrm{NO}_{3}\right)_{2} \cdot 6 \mathrm{H}_{2} \mathrm{O}$ como sal precursor. Após a impregnação, os materiais obtidos foram calcinados sob fluxo de ar, de acordo com o seguinte procedimento: (i) aquecimento da temperatura ambiente até $673 \mathrm{~K}$ (taxa de aquecimento $=0,5 \mathrm{~K} / \mathrm{min}$ ), (ii) aquecimento de 673 até $1073 \mathrm{~K}$, usando-se uma taxa de aquecimento de $2 \mathrm{~K} / \mathrm{min}$. 
A área específica foi avaliada pelo método BET através da adsorção de nitrogênio a 77 K, utilizando o equipamento ASAP 2020 (Micromeritics).

As análises de difração de raios X (DRX) foram feitas utilizando um difratômetro Rigaku modelo Miniflex, com radiação $\mathrm{CuK} \alpha$, entre $2 \theta=25^{\circ}$ a $80^{\circ}$, usando-se um passo de $0.04^{\circ}$ e um tempo de contagem de 1 segundo por passo. Para o cálculo do tamanho de cristalito do $\mathrm{Ni}^{0}$ e do suporte após a redução, foi feita a passivação seguida de análises de DRX. A passivação foi feita de acordo com o procedimento previamente descrito na literatura (da Silva et. al., 2013).

As análises de redução a temperatura programada (TPR) foram realizadas em uma unidade acoplada a um detector de condutividade térmica. Foi feito um aquecimento da temperatura ambiente até $1273 \mathrm{~K}$ a uma taxa de $10 \mathrm{~K} / \mathrm{min}$, utilizando-se uma mistura de $2 \%$ de $\mathrm{H}_{2}$ em $\mathrm{N}_{2}$ com vazão de $30 \mathrm{ml} / \mathrm{min}$.

As análises termogravimétricas das amostras, após a reação, foram realizadas em um equipamento TA Instruments (SDT Q600) para determinar a quantidade de carbono formada na superfície dos catalisadores. $O$ procedimento consiste no aquecimento de aproximadamente $10 \mathrm{mg}$ de amostra sob fluxo de ar da temperatura ambiente até $1273 \mathrm{~K}$, com taxa de aquecimento de $20 \mathrm{~K} / \mathrm{min}$, monitorando a variação de massa.

A reação de reforma seca do metano foi realizada em um reator de quartzo, a pressão atmosférica. O catalisador foi diluído em $\mathrm{SiC}$ (colocar a razão SiC/massa de catalisador) com o intuito de evitar pontos quentes. As amostras foram reduzidas sob $\mathrm{H}_{2}$ puro a $1023 \mathrm{~K}$, por 72 min. A reação foi, então, realizada a $1073 \mathrm{~K}$, usando-se uma razão molar de $\mathrm{CH}_{4} / \mathrm{CO}_{2}=1,0$.

\section{RESULTADOS E DISCUSSÕES}

Todos os suportes apresentaram valores baixos de área específica $(\mathrm{CeZr}: 39 \mathrm{~m} / \mathrm{g}$; CeNb: $22 \mathrm{~m}^{2} / \mathrm{g}$ e CePr: $18 \mathrm{~m}^{2} / \mathrm{g}$ ), assim como foi observado na literatura para óxidos a base de Ce calcinados em temperaturas altas (Augusto, 2012). Em relação ao dopante, o CeZr é o que apresentou maior área específica, enquanto que o CePr mostrou o menor valor. Trabalhos na literatura (Kaspar et al., 1999) sugerem que a substituição de átomos de Ce por $\mathrm{Zr}$ na estrutura do óxido de cério promove um aumento na estabilidade térmica do material e evita sua sinterização.

Os resultados das análises de DRX dos suportes e dos catalisadores calcinados são apresentados nas Figuras 1a e 1b, respectivamente. Os difratogramas dos suportes CeZr e CePr apresentaram as linhas de difração correspondentes às soluções sólidas do cério com seu respectivo dopante, ou seja, $\mathrm{Ce}_{0,9} \mathrm{Zr}_{01} \mathrm{O}_{1,95}$ (ICSD 152478), $\mathrm{Ce}_{0,9} \mathrm{Pr}_{0,1} \mathrm{O}_{2}$ (ICSD182184). O teor de $\mathrm{Nb}$ usado foi superior ao seu limite de solubilidade em $\mathrm{CeO}_{2}(1,4 \%$ em peso) (RamirezCabrera et al., 2002). Entretanto, não foram detectadas as linhas relativas ao $\mathrm{Nb}_{2} \mathrm{O}_{5}$ ou $\mathrm{CeNbO}_{4}$. Nos difratogramas dos catalisadores calcinados, além das linhas correspondentes ao suporte, foram observadas as linhas de difração características do NiO.

Ao observar os diâmetros de cristalitos dos suportes calcinados a $1073 \mathrm{~K}$ (Tabela 1), é possível verificar que a amostra contendo $\mathrm{Zr}$ como dopante apresentou o menor tamanho de cristalito de cério, o que está de acordo com os valores de área específica. Esse resultado 
indica que esta amostra teve uma maior resistência à sinterização durante a calcinação do material.

(a)

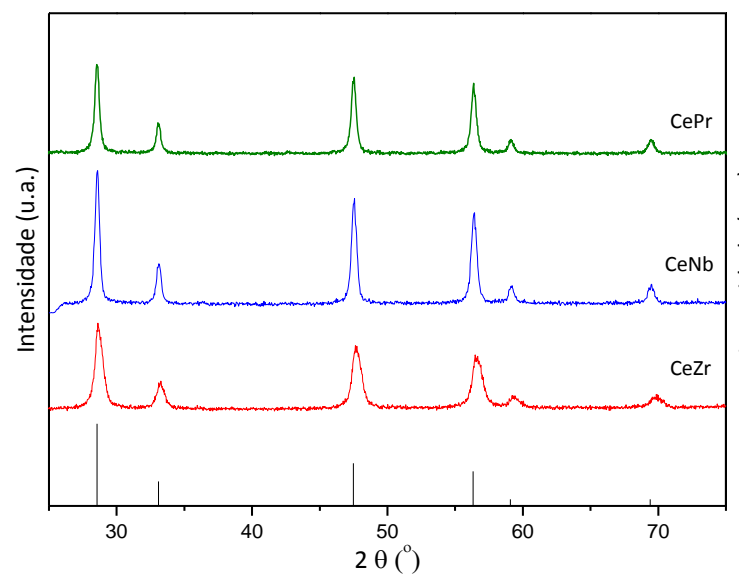

(b)

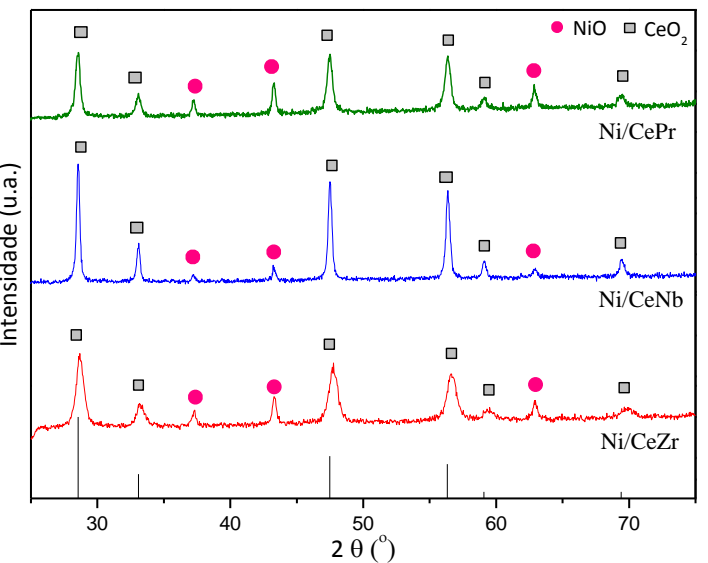

Figura 1: Difratogramas dos (a) suportes e (b) catalisadores, após a calcinação.

Em relação aos catalisadores (Tabela 1), nota-se que o material dopado com $\mathrm{Zr}$, mais uma vez, foi o que apresentou menor diâmetro de cristalito do $\mathrm{CeO}_{2}$ enquanto que os catalisadores $\mathrm{Ni} / \mathrm{CeNb}$ e $\mathrm{Ni} / \mathrm{CePr}$ apresentaram o mesmo valor. Além disso, é possível observar que no material contendo $\mathrm{Zr}$, a impregnação de níquel não acarretou mudanças significativas no tamanho de cristalito do suporte. Já nos materiais contendo $\mathrm{Nb}$ e $\mathrm{Pr}$, a impregnação do metal levou a maiores diâmetros de cristalito de $\mathrm{CeO}_{2}$. Em relação ao $\mathrm{NiO}$, foi observado que o material contendo $\mathrm{Nb}$ apresentou o maior tamanho de cristalito.

Tabela 1 - Valores de tamanho de cristalito do $\mathrm{CeO}_{2}$ e $\mathrm{NiO}$ (calculados através da equação de Scherrer) para os suportes e catalisadores calcinados a 1073K.

\begin{tabular}{|c|c|c|}
\hline \multirow{2}{*}{ Amostras } & \multicolumn{2}{|c|}{ Tamanho de Cristalito (nm) } \\
\cline { 2 - 3 } & ${ }^{\mathrm{a}} \mathrm{CeO}_{2}$ & ${ }^{\mathrm{b}} \mathrm{NiO}$ \\
\hline $\mathbf{C e Z r}$ & 13 & - \\
\hline $\mathbf{C e N b}$ & 22 & - \\
\hline $\mathbf{C e P r}$ & 21 & - \\
\hline $\mathbf{N i} / \mathbf{C e Z r}$ & 12 & 24 \\
\hline $\mathbf{N i} / \mathbf{C e N b}$ & 26 & 30 \\
\hline $\mathbf{N i} / \mathbf{C e P r}$ & 26 & 22 \\
\hline
\end{tabular}

${ }^{\mathrm{a}}$ calculado usando o plano (111) do $\mathrm{CeO}_{2} ;{ }^{\text {b }}$ calculado usando o plano (111) do $\mathrm{NiO}$

Nos difratogramas das amostras reduzidas e passivadas (não apresentados) foram observadas apenas as linhas de difração dos suportes e do níquel metálico. Analisando-se os valores de tamanho de cristalito do níquel metálico (calculados através da equação de Scherrer, usando-se o plano (111) do $\mathrm{Ni}^{0}$ ), observa-se que o material contendo $\mathrm{Zr}$ como dopante apresentou o menor valor de diâmetro de cristalito $(24 \mathrm{~nm})$. Além disso, os materiais 
suportados em $\mathrm{Nb}$ e $\mathrm{Pr}$ apresentaram valores de tamanho de cristalito de $\mathrm{Ni}^{0}$ bem similares (32 e $33 \mathrm{~nm}$, respectivamente).

Os perfis de TPR dos suportes, dos catalisadores e do óxido de níquel podem ser observados na Figura 2. Os resultados mostram que o óxido de níquel apresenta um pico de redução a $719 \mathrm{~K}$ e um ombro a $770 \mathrm{~K}$. No caso dos catalisadores a base de CeZr e CePr foram observados perfis de redução similares com picos de redução entre 726-757 K e um ombro entre 805-890 K. De acordo com a literatura (Passos et al., 2005), o perfil de redução do óxido de cério apresenta um pequeno consumo de $\mathrm{H}_{2}$, em torno de $820 \mathrm{~K}$, e um maior consumo a $1220 \mathrm{~K}$. O consumo em temperaturas mais baixas é atribuído à redução do $\mathrm{CeO}_{2}$ superficial, enquanto que o consumo em maiores temperaturas está relacionado à redução do $\mathrm{CeO}_{2}$ mássico. Quando dopantes como o $\mathrm{Zr}$ são inseridos na estrutura do $\mathrm{CeO}_{2}$, observa-se apenas um pico de redução em temperaturas em torno de 840-860 K, sugerindo que a presença do $\mathrm{Zr}$ promove a redução do óxido de cério mássico. Isso é atribuído ao aumento da mobilidade de oxigênio do $\mathrm{CeO}_{2}$ devido à formação da solução sólida (Passos et al., 2005). Além disso, a adição do metal ao suporte também promove a redução do óxido misto.

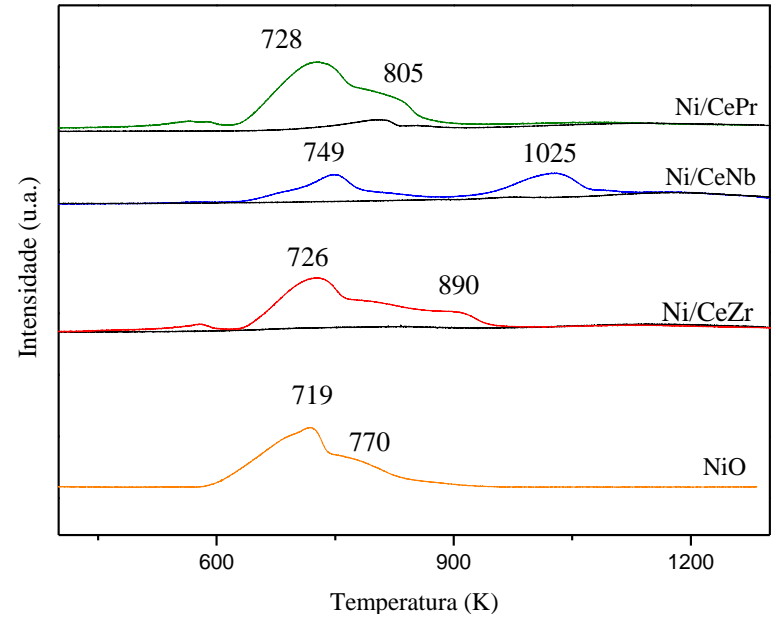

Figura 2 - Perfis de TPR das amostras Ni/CePr, Ni/CeNb, Ni/CeZr e NiO.

Assim, neste trabalho, o consumo de hidrogênio observado nos perfis de TPR das amostras $\mathrm{CeZr}$ e $\mathrm{CePr}$ pode ser atribuído à redução do $\mathrm{NiO}$ mássico juntamente com a redução do $\mathrm{CeO}_{2}$ promovida pela adição do dopante e do metal. A semelhança entre os perfis de redução dos catalisadores com o perfil de redução do $\mathrm{NiO}$ mássico sugere a formação de grandes partículas de óxido de níquel nessas amostras, o que está de acordo com os resultados de DRX. Esse resultado indica que não há uma forte interação entre o $\mathrm{NiO}$ e o suporte (Augusto, 2012). Além disso, comparando os perfis de redução das amostras a base de CeZr e CePr, observa-se que a amostra contendo $\mathrm{Zr}$ apresenta um maior consumo em temperaturas mais altas. Esse resultado indica que o efeito promotor da redução do $\mathrm{CeO}_{2}$ foi mais significativo na amostra contendo $\mathrm{Pr}$, o que sugere uma maior redutibilidade e, consequentemente, uma maior capacidade de armazenamento de oxigênio para essa amostra. No caso do catalisador $\mathrm{Ni} / \mathrm{CeNb}$, além do consumo de hidrogênio correspondente à redução do $\mathrm{NiO}$, observou-se um pico de redução em uma temperatura $(1025 \mathrm{~K})$ superior à encontrada nos outros catalisadores, que poderia ser atribuído à redução de óxido de cério mássico e do 
óxido de nióbio. Esses resultados indicam que não foi verificada uma promoção significativa da redução do suporte neste catalisador, sugerindo que a solução sólida não foi formada e que, consequentemente, ocorreu a segregação do $\mathrm{Nb}$. O óxido de nióbio não estaria sendo observado nas análises de DRX, devido ao seu baixo teor.

A Figura 3 mostra a conversão do $\mathrm{CH}_{4}$ e $\mathrm{CO}_{2}$ e as seletividades para $\mathrm{H}_{2}$ e $\mathrm{CO}$ na reforma seca do metano a $1073 \mathrm{~K}$, nos catalisadores de $\mathrm{Ni} / \mathrm{CeNb}, \mathrm{Ni} / \mathrm{CePr}$ e $\mathrm{Ni} / \mathrm{CeZr}$. O catalisador $\mathrm{Ni} / \mathrm{CeZr}$ apresentou os maiores valores de conversão inicial de metano e de $\mathrm{CO}_{2}$. Foi observada uma pequena queda na conversão de metano e $\mathrm{CO}_{2}$ no início da reação para os catalisadores Ni/CeZr e Ni/CePr. Entretanto, após esse período inicial de desativação, esses catalisadores mantiveram-se praticamente estáveis ao longo da reação. Para o catalisador $\mathrm{Ni} / \mathrm{CeNb}$, foi observada uma queda suave e contínua durante a reação. Com relação à seletividade para $\mathrm{H}_{2}$ e $\mathrm{CO}$, todos os materiais apresentaram valores de seletividade similares, sendo de 60-65 \% e de 35-40\% para $\mathrm{CO}$ e $\mathrm{H}_{2}$, respectivamente. Esses valores correspondem a uma razão $\mathrm{H}_{2} / \mathrm{CO}$ em torno de $0,54-0,67$. Esse valor abaixo de 1,0 pode ser atribuído à ocorrência da reação reversa de deslocamento de água.
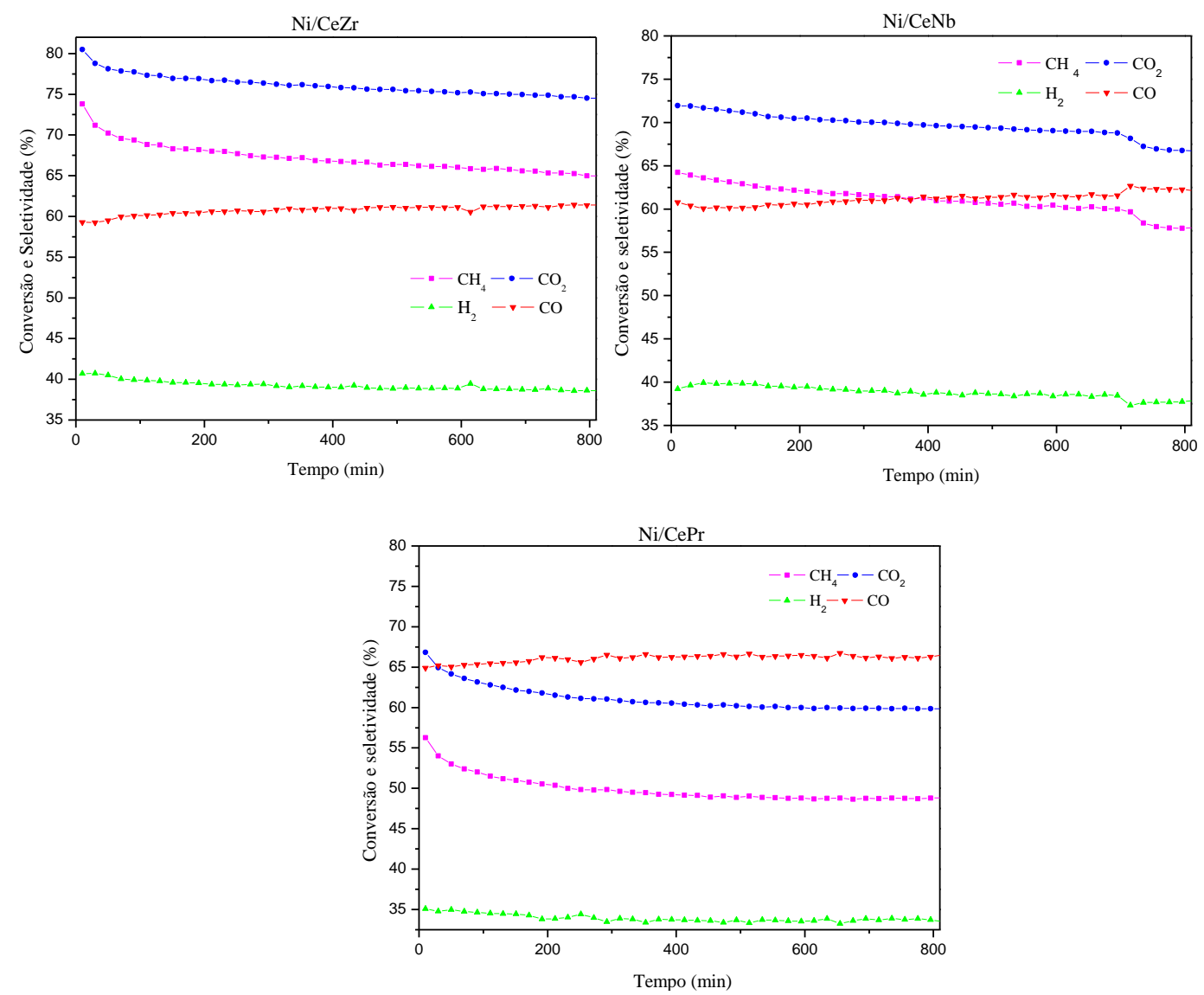

Figura 3- Valores de conversão de metano e $\mathrm{CO}_{2}$ e de seletividade para $\mathrm{H}_{2}$ e $\mathrm{CO}$ obtidos para todos os catalisadores durante a reforma seca do metano a $1073 \mathrm{~K}$.

Com o objetivo de avaliar a natureza e a quantidade de carbono formada durante a reação, foram realizadas análises termogravimétricas. Os perfis de TPO obtidos para todos os catalisadores são apresentados na Figura 4. Para o catalisador Ni/CeZr, foi observado apenas 
um pico de oxidação em torno de $900 \mathrm{~K}$. No caso do catalisador $\mathrm{Ni} / \mathrm{CeNb}$, foi detectado um pico a $913 \mathrm{~K}$ e um ombro a $765 \mathrm{~K}$. Com relação ao perfil de TPO do catalisador CePr, não foi observada nenhuma oxidação significativa. De acordo com a literatura (Silva et al, 2012), os picos acima de $773 \mathrm{~K}$ correspondem à oxidação de nanotubos de carbono com paredes simples e com paredes múltiplas (SWNT e MWNT), enquanto que os picos em temperaturas acima de $973 \mathrm{~K}$ estariam relacionados à oxidação de grafite. Assim, os resultados obtidos neste trabalho indicam a presença de ambos os tipos de filamentos de carbono (SWNT e MWNT) nos catalisadores a base de CeZr e CeNb.

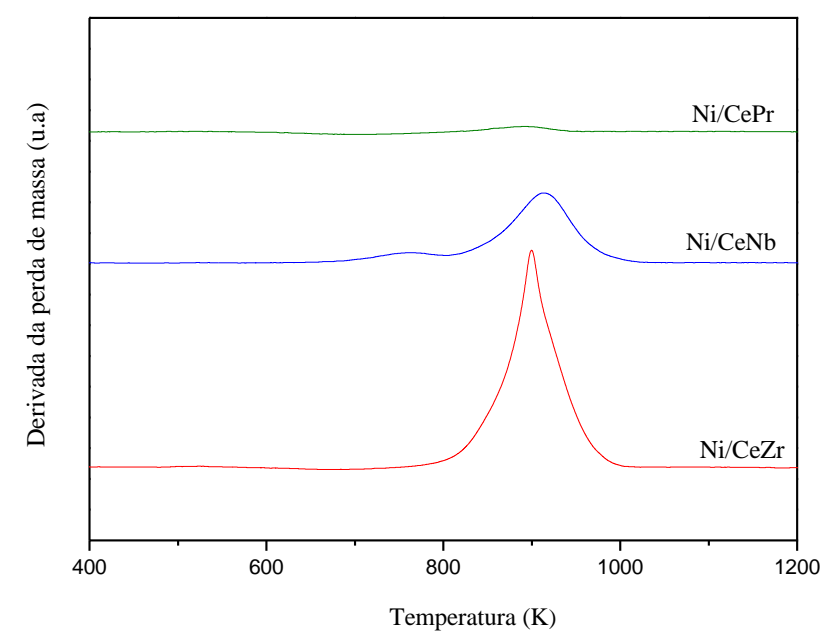

Figura 4 - Perfis de TPO obtidos, após a reação, para todos os catalisadores

$\mathrm{O}$ catalisador de $\mathrm{Ni} / \mathrm{CePr}$ foi o que apresentou a menor quantidade de carbono formada (0,4 $\mathrm{mg} \mathrm{C} /\left(\mathrm{g}_{\text {cat.h}}\right)$ ), enquanto que o $\mathrm{Ni} / \mathrm{CeNb}$ e o $\mathrm{Ni} / \mathrm{CeZr}$ apresentaram 12,1 e $24,2 \mathrm{mg}$ $\mathrm{C} /\left(\mathrm{g}_{\text {cat.h}} \mathrm{h}\right)$ respectivamente. Na literatura (Stagg-Williams et al., 2000) foi proposto um mecanismo de formação de carbono sobre catalisadores de metálicos suportados durante a reforma seca do metano, envolvendo duas etapas. Na primeira etapa ocorre a dissociação do carbono sobre o cristalito metálico, produzindo hidrogênio e espécies de carbono altamente reativas. Na segunda etapa, ocorre a reação do carbono com o oxigênio proveniente do suporte. $\mathrm{O}$ oxigênio é formado durante a adsorção dissociativa do $\mathrm{CO}_{2}$ e reoxidação do suporte. Assim, suportes com alta capacidade de armazenamento de oxigênio, como óxidos mistos a base de cério, promovem a remoção do carbono da superfície do cristalito de níquel. Neste trabalho, os resultados obtidos nas análises de TPR sugerem que o catalisador a base de CePr apresenta uma maior redutibilidade, o que indica uma maior mobilidade de oxigênio, favorecendo a remoção do carbono da superfície metálica.

\section{CONCLUSÃO}

Os resultados obtidos mostraram que o catalisador $\mathrm{Ni} / \mathrm{CeZr}$ apresentou a maior atividade inicial durante a reforma seca do metano, devido ao menor tamanho de cristalito de $\mathrm{Ni}$ metálico, conforme observado nas análises de DRX. Entretanto, a menor formação de carbono foi observada para o catalisador a base de CePr. Esse resultado foi atribuído a sua maior capacidade de armazenamento de oxigênio (como revelado pelas análises de TPR), que estaria promovendo o mecanismo de remoção do carbono da superfície do níquel metálico. 


\section{AGRADECIMENTOS}

Os autores gostariam de agradecer a CAPES e ao CNPq pelas bolsas concedidas as alunas Renata Fonseca e Andressa da Silva.

\section{REFERÊNCIAS}

AHMED, S.; KRUMPELT, M. Hydrogen from hydrocarbon fuels cells. Int. J. of H. En., v. 26, p. 291-01, 2001.

AUGUSTO, B. L. Desenvolvimento de ânodos para geração de energia em células a combustível do tipo SOFC alimentadas com bio-etanol. Dissertação de Mestrado, Universidade Federal Fluminense, 2012.

DA SILVA, A. A. A.; DA COSTA, L. O. O.; MATTOS, L. V.; NORONHA, F. B. The study of the performance of Ni-based catalysts obtained from $\mathrm{LaNiO}_{3}$ perovskite-type oxides synthesized by the combustion method for the production of hydrogen by reforming of ethanol. Catal. Today, v. 213, p. 25-32, 2013.

JASINSKI, P.; SUZUKI, T.; PETROVSKY, V.; ANDERSONB H.U. Nanocomposite Nickel Ceria Cermet with Low Nickel Content for Anode-Supported SOFCs. Electrochem. and Solid-State Lett, v. 8, p. A 219, 2005.

KASPAR, J.; FORNASIERO, P.; GRAZIANI, M. Use of $\mathrm{CeO}_{2}$ based oxides in the three-way catalysis. Catalysis Today, v. 50, p. 285-298, 1999.

KE, K.; GUNJI, A.; MORI, H.; TSUCHIDA, S.; TAKAHASHI, H.; UKAI, K.; MIZUTANI, Y.; SUMI, H.; YOKOYAMA, M.; Waki, K. Effect of oxide on carbon deposition behavior of $\mathrm{CH} 4$ fuel on Ni/ScSZ cermet anode in hihh temperature SOFCs. S. S. Ion., v. 177, p. 541-547, 2006.

MATTOS, L.V.; de OLIVEIRA, E.R.; RESENDE, P.D.; NORONHA, F.B.; PASSOS, F.B. Partial oxidation of methane on Pt/Ce- $\mathrm{ZrO}_{2}$ catalysts. Catal. Today, v. 77, p. 245, 2002.

MODAFFERRI, V.; PANZERA, G.; BAGLIO, V.; FRUSTRI, F.; ANTONUCCI, P.L. Propane reforming on $\mathrm{Ni}-\mathrm{Ru} / \mathrm{GDC}$ catalyst: $\mathrm{H} 2$ production for IT-SOFCs under SR and ATR conditions. Ap. Catal. A, v. 334, p. 1-9, 2008.

PASSOS, F.B.; de OLIVEIRA, E.R.; MATTOS, L.V.; NORONHA, F.B. Partial oxidation of methane to synthesis gas on $\mathrm{Pt} / \mathrm{Ce} x \mathrm{Zr}_{1-} x \mathrm{O}_{2}$ catalysts: the effect of the support reducibility and of the metal dispersion on the stability of the catalysts. Catal.Today, v. 101, p. 23-30, 2005. SILVA, A.A.A. Tese de Mestrado, Universidade federal fluminense, 2012.

RAMIREZ-CABRERA, E., ATKINSON, A., CHADWICK, D., Reactivity of ceria, Gd- and Nb-doped ceria to methane, Catal. Today v. 36, p. 193-206, 2002.

SUN, C.; STIMMING, U. J. Recent anode advances in solid oxide fuel cells. J. of P. Source., v. 171, p. 247, 2007.

STAGG-WILLIAMS, S.M.; NORONHA, F.B.; FENDLEY, G.; RESASCO, D.E. $\mathrm{CO}_{2}$ Reforming of $\mathrm{CH}_{4}$ over $\mathrm{Pt} / \mathrm{ZrO}_{2}$ Catalysts Promoted with La and Ce Oxides. J. Catal, v. 194, p. 240, 2000. 